\title{
The Performance Evaluation of Adaptive Guard Channel Scheme in Wireless Network
}

\author{
O. A. Ojesanmi ${ }^{\text {a,* }}$, O. A. Lawal ${ }^{\text {b }}$, F. T. Ibharalu ${ }^{\mathrm{a}}$, I. A. Adejumobi ${ }^{\mathrm{c}}$ \\ ${ }^{a}$ Department of Computer Science, Federal University of Agriculture, Abeokuta, Ogun State, NIGERIA. \\ ${ }^{b}$ Department of Computer Science, Moshood Abiola Polytechnic Ojere, Abeokuta, Ogun State, NIGERIA. \\ ${ }^{c}$ Department of Electrical / Electronics Engineering, Federal University of Agriculture, Abeokuta, Ogun State, \\ NIGERIA.
}

\begin{abstract}
Dynamic Guard Channels (DCG) reduces the dropping and blocking rates in a network. However, most of the existing DGC allocations are not quite efficient because there were consideration for only the Handoff (HO) calls while the New calls (NC) were not considered; this leads to poor Quality of Service (QoS) for NC. Although it is better to give priority to $\mathrm{HO}$ calls over NC since the breaking of the connection of an established communication is more annoying than blocking a NC. Thus, there is need to provide an alternative approach that guarantees an acceptable QoS in terms of both the HC and the NC. This paper presents the performance evaluation of an adaptive guard channel allocation; the scheme made use of two different models (1) guard channel with fuzzy logic (2) guard channel without fuzzy logic. Priority is given to handoff call due to the scarcity of radio spectrum. When all the guard channels have been allocated and the arrival rate of handoff calls keeps on increasing, new set of threshold values would be estimated by fuzzy logic model. Performance metrics are; Call Blocking Rate (CBR), Call Dropping Rate (CDR) and Throughput. Results showed that guard channel with fuzzy logic has the CBR values range from $24.02 \%$ to 69.015 and CDR values range from 12.025 to $18.90 \%$ while guard channel without fuzzy logic has CBR values range from $28.22 \%$ to $75.65 \%$ and CDR values range from $19.06 \%$ to $36.50 \%$. The scheme proved to be more efficient in congestion control in wireless network.
\end{abstract}

Keywords: handoff call, new call, buffer, call dropping probability, arrival rate, throughput

\section{INTRODUCTION}

The Global System for Mobile (GSM) communication has become so important in the life of man and the use of the GSM is not being limited to the rich alone but to all human beings be it rich, poor, old or young. This has led to the ever-growing demand for GSM communications by people as reported by the GSM Association which could make congestion inevitable [1,2]. Cellular networks have evolved into one of the most exciting areas in telecommunications industries. New generations of cellular networks support a wide variety of services such as voice, data, and video. The user of $3.5 \mathrm{G}$ or newer cellular networks can make a voice call or/ and make data connection, while watching an online movie. Mobile devices are becoming more and more indispensable in our daily life. Technological advancements and the availability of high-speed connections have made the mobile device a handy tool for managing day-to-day tasks

* Corresponding author (Tel: +234 (0)805 605 2007)

Email addresses: dejioje@yahoo.com (O. A.

Ojesanmi), oyindamola2008@gmail.com (O. A. Lawal), ibharaluft@yahoo.co.uk (F. T. Ibharalu),

engradejumobi@yahoo.com (I. A. Adejumobi) involving sensitive data, monetary transactions etc [3]. People show more interest in digital payment, mobile banking, digital news; this led to exponential growth and tremendous increase in mobile users which put pressure on bandwidth.

In the past, existing mobile devices were not equipped to connect to more than one access network simultaneously. However, in recent times, mobile devices are now equipped to physically connect to multiple data links. With this development, hand-over process occurs frequently and simultaneously, operators therefore need to increase their network capacity to provide efficient network services to meet this growing demand [4]. Many factors like quality of service (QoS), lower power usages and ever-present mobile coverage play important role to select the mobile operator by the users. Thus, wireless networks should be designed with desired quality-of-service requirements to achieve a better performance.

A new adaptive channel allocation method based on guard channels was proposed, fuzzy logic model is applied to determine the threshold set as guard channels in the base station. The proposed channel allocation scheme helps to give priority to the handoff call over the new call and also reduce 
congestion in mobile network.

\section{LITERATURE REVIEW}

Channel assignment technique is basically of three major types: Fixed channel assignment (FCA), Dynamic channel assignment (DCA) and Hybrid channel assignment (HCA). In FCA schemes, the cellular area is partitioned into a number of cells and a number of channels assigned are static. It is simple but it is not easy to adapt to the changes in traffic conditions. Thus, DCA schemes have been introduced in order to overcome those challenges of FCA schemes. In DCA, all channels are placed in a central pool and they are accessible to all cells as needed. DCA schemes is flexible and it also adapt easily to the traffic condition. Although DCA performs better than FCA under high to moderate traffic but its performance is worse than that of FCA under conditions of heavy traffic changes. HCA scheme is a combination of both FCA and DCA techniques, where channels are grouped into two sets; one set of channels is statically assigned to a base station as in FCA and the second set is placed in a central pool, assigned in a DCA manner [5-7].

The mobile management or the quality-of service (QoS) for a cellular communication is a function of handoff process. The said process could be defined as the call transfers of a mobile station from one base station to another base station or one cell boundary to another cell boundary or from one base station system [8]. Handoff refers to the process by which an active mobile station changes its point of attachment to the network, or when such a change is attempted. The handover process is one of the essential means that guarantees user mobility in a mobile communication network. Handoff is a key element in wireless cellular networks. Numerous protocols have been designed to limit the probability of interrupting ongoing calls in a situation whereby the call termination of the on-going calls is becoming difficult to avoid. Different ideas and approaches are proposed to reduce the handover dropping probability. One approach to reduce the handover failure rate is to prioritize handover call over new calls [9]. Handover prioritization schemes have a significant impact on the call dropping probability and call blocking probability. Such scheme permits high utilization of bandwidth while guaranteeing the quality of service of handover calls. Some of these handover prioritization schemes are; guard channel prioritization scheme, call admission control prioritization scheme, handover queuing prioritization scheme, cell overlapping and load balancing scheme.

Existing scheme such as [10] presented an adaptive call admission control and resource allocation in multi server wireless/cellular network. The proposed mechanism did not a controller that can optimally allocate the ordinary channels and guard channels in each cell of the cluster in cellular network. [11] did a performance comparison of dynamic guard channel assignment with buffered prioritized scheme for mobile WiMAX network.
While the prioritized guard channel assignment scheme with buffer reduced handover call dropping probability more than any other channel assignment scheme, it compromise the QoS of new call as it led to a higher call blocking probability [12] developed efficient utilization of channels using dynamic guard channel allocation with channel borrowing strategy in handoffs. The scheme only gave priority to the handoff call and did not considered new call. [13] designed a channel allocation scheme for cellular network using fuzzy logic. The scheme only considered centroid defuzzification method, comprehensive simulation and analysis could be carried out to investigate other forms of defuzzification such as center of gravity (COG), mean of maximum (MOM), and center average methods. [14] designed an improved channel allocation scheme for mobile cellular network. The work applied hybrid channel allocation method which gives better performance, majorly under heavy traffic loads. [15] developed a fuzzy-based dynamic time slot allocation for wireless body area networks. The model only considered centroid defuzzification method, the result obtained can be compared with other methods such as center of gravity (COG), mean of maximum (MOM), and center average methods. [16] presented a cellular cluster channel allocation using an edge weight frequency assignment algorithm. The scheme should be enhanced further to improve better network optimization. [17] Presented a review work on different models of fixed channel assignment in mobile communication systems. The survey work did not indicate the best fixed channel allocation scheme in cellular mobile network. [18] designed a resource allocation algorithm for LTE networks using fuzzy based adaptive priority and effective bandwidth estimation.

\section{MODEL DESCRIPTION}

In this paper, the model made use of adaptive channel assignment scheme that consists of adaptive guard channels and opened channels. The guard channels represent the frequency reserved exclusively for handoff calls in order to continue to sustain the established communication. The opened channels are accessible to both the handoff calls and the new calls that arrived in the base station. The channel assignment algorithm setup a specific threshold value, "g", that is equivalent to the number of the guard channels used at different instances. When all the guard channels have been allocated, the newly arrived handoff calls will be dropped. At this point, when there is higher arrival rate of handoff calls than the set threshold value, another "g" would be computed.

The work considered two different schemes; an adaptive channel assignment scheme with fuzzy logic model and an adaptive channel assignment scheme without fuzzy logic model. The scheme with fuzzy logic model made use of fuzzy logic system to estimate the number of guard channels as it output value, taking into consideration the total number of handoff calls in the guard channels and 
the number of free channels as the inputs into the fuzzy system. The system architecture diagram is represented with Fig. 1 while the system flow chart is represented with Fig. 2.
Algorithm 1
Start
Call Arrival
IF (Call arrived $=$ New Call) THEN
"Check for Opened Channel Available"
IF (Opened Channel is Available) THEN "Accept Call"
ELSE
"Block Call"
END IF
ELSE IF (Call arrived = Hand-off Call) THEN
"Check for Opened Channel Available"
IF (Opened Channel is Available) THEN "Accept Call"
ELSE IF (Opened Channels are not Available) "Check for any Free Guard Channel"
IF (Any Free Guard Channel is found) THEN "Accept Call"
ELSE

"Apply Fuzzy Logic System to get new Threshold"

IF (Guard Channel is found) THEN "Accept Call"

ELSE

"Drop Call"

\section{END IF}

END IF

END IF

\subsection{Fuzzy Logic System (FLS)}

Fuzzy Logic System is a problem-solving control system methodology that lends itself to implementation in systems based upon ambiguous, or imprecise input information. The FLS approach in solving problems mimics how a person would make decisions, much faster. FLS incorporates a simple, rule-based IF X AND Y THEN Z approach to solving control problem rather than attempting to model a system mathematically. FLS make use of linguistic variable and terms such as HIGH and LOW. The fuzzy rules used by the interference engine is indicated in Table 1.

Table 1: Fuzzy Rule Base.

\begin{tabular}{lcc}
\hline $\begin{array}{l}\text { Total Free } \\
\text { Channel }\end{array}$ & $\begin{array}{c}\text { Number of } \\
\text { handoff calls in } \\
\text { GC }\end{array}$ & $\begin{array}{c}\text { Threshold/Guard } \\
\text { channel }\end{array}$ \\
\hline $\mathrm{H}_{F C}$ & $\mathrm{H}_{h o}$ & $\mathrm{H}$ \\
$\mathrm{L}_{F C}$ & $\mathrm{H}_{h o}$ & $\mathrm{H}$ \\
$\mathrm{H}_{F C}$ & $\mathrm{~L}_{h o}$ & $\mathrm{H}$ \\
$\mathrm{L}_{F C}$ & $\mathrm{~L}_{h o}$ & $\mathrm{~L}$ \\
\hline
\end{tabular}

FREE CHANNEL (HIGH $=\mathrm{H}_{F C}$ AND LOW $=\mathrm{L}_{F C}$ ) GUARD CHANNEL HANDOFF NUMBER (HIGH= $\mathrm{H}_{G c h o N}$, AND LOW $\left.=\mathrm{L}_{G c h o N}\right)$

THRESHOLD/GUARD CHANNEL (HIGH = H AND LOW $=\mathrm{L}$ )

\section{ASSUMPTION AND NOTATIONS}

In this work, we developed a Fuzzy Logic Model (FLM) to control congestion in mobile communica- tion system. There are few assumptions and notations that we have considered: the model deals with a network with homogeneous cells, therefore what applies in one cell is applicable in all other cells in the mobile network. We considered for each base station with allocation of total channels " $t$ ", that guard " $g$ " is reserved exclusively for handoff calls. Also, we assumed that both the handoff calls and the new/originating calls arrive according to Poisson arrivals. Parameters were used under the assumption that all the channels in the base station have passed the constraint test: Co-Channel Constraint, Adjacent Channel Constraint and Co-Site Constraint. The parameters are defined as follows: $\lambda_{h}$ - Arrival rate for handoff calls.

$\lambda_{n}-$ Arrival rate for new calls.

$\mu$-Service rate i.e. the rate at which requests are serviced. $\mathrm{C}_{t}-$ Total number of channels in a base station.

Let $\mathrm{X}$ be a set. A fuzzy subset $\mathrm{A}$ of $\mathrm{X}$ is characterized by a membership function. fa: $\mathrm{X}[0,1]$. The membership function is equivalent to the identity function of a classical set.

"In fuzzy sets, each element is mapped to $[0,1]$, by membership function. $\mu \mathrm{A}: \mathrm{X} \rightarrow[0,1]$ where $[0,1]$ means real numbers between 0 and 1 (including 0 and 1 ). The system performance is measured by using two major parameters such as call blocking rate and call dropping rate. The two parameters are defined as shown in Eq. (1) and (2).

$$
\begin{gathered}
\text { Call Blocking Rate }= \\
\frac{\text { Total number of calls blocked }}{\text { Total number of new calls initiated }}
\end{gathered}
$$

Call Dropping Rate $=$

Total number of calls dropped

Total number of handoff calls

Throughput: Throughput is the actual amount of data that is successfully sent/received over the communication link.

\section{RESULTS AND DISCUSSION}

A simulation model was used to evaluate the performance of the proposed scheme. Figure 3 shows the result of the call dropping rate (CDR) without the use of fuzzy logic model, with the CDR values that range from 19.06 to 36.50 . Also, the call dropping rate (CDR) with the use of fuzzy logic model, with the CDR values that range from 12.02 to 18.90 . The combination of guard channel allocation scheme together with the fuzzy logic model has therefore proved to be more efficient with a far lower CDR. Therefore, there is an improved performance result in terms of reduced call dropping rate with the application of fuzzy logic model. Thus, the proposed scheme with fuzzy logic model has proved to be more efficient than that of the ordinary guard channel scheme. The 


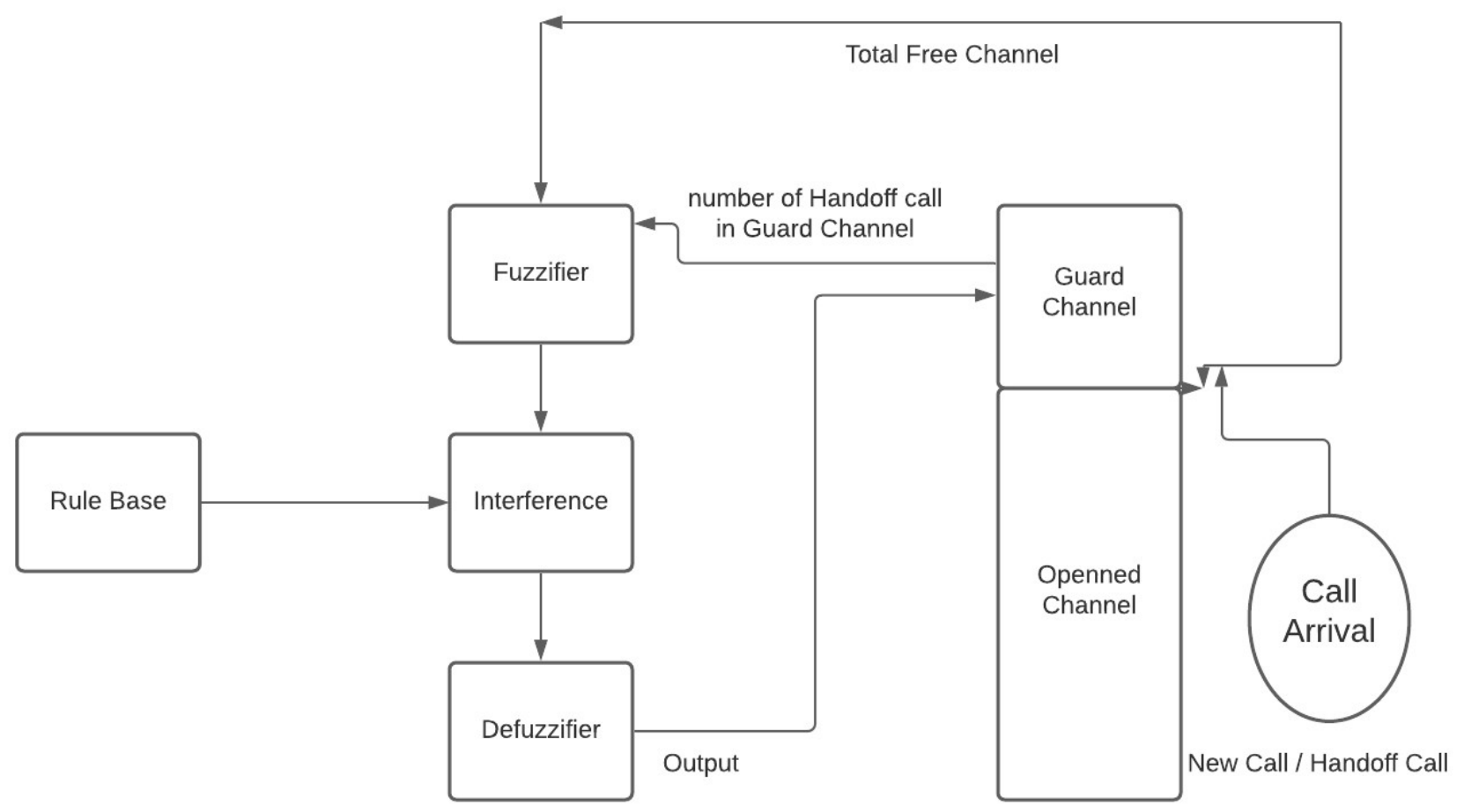

Figure 1: System Architecture Diagram.

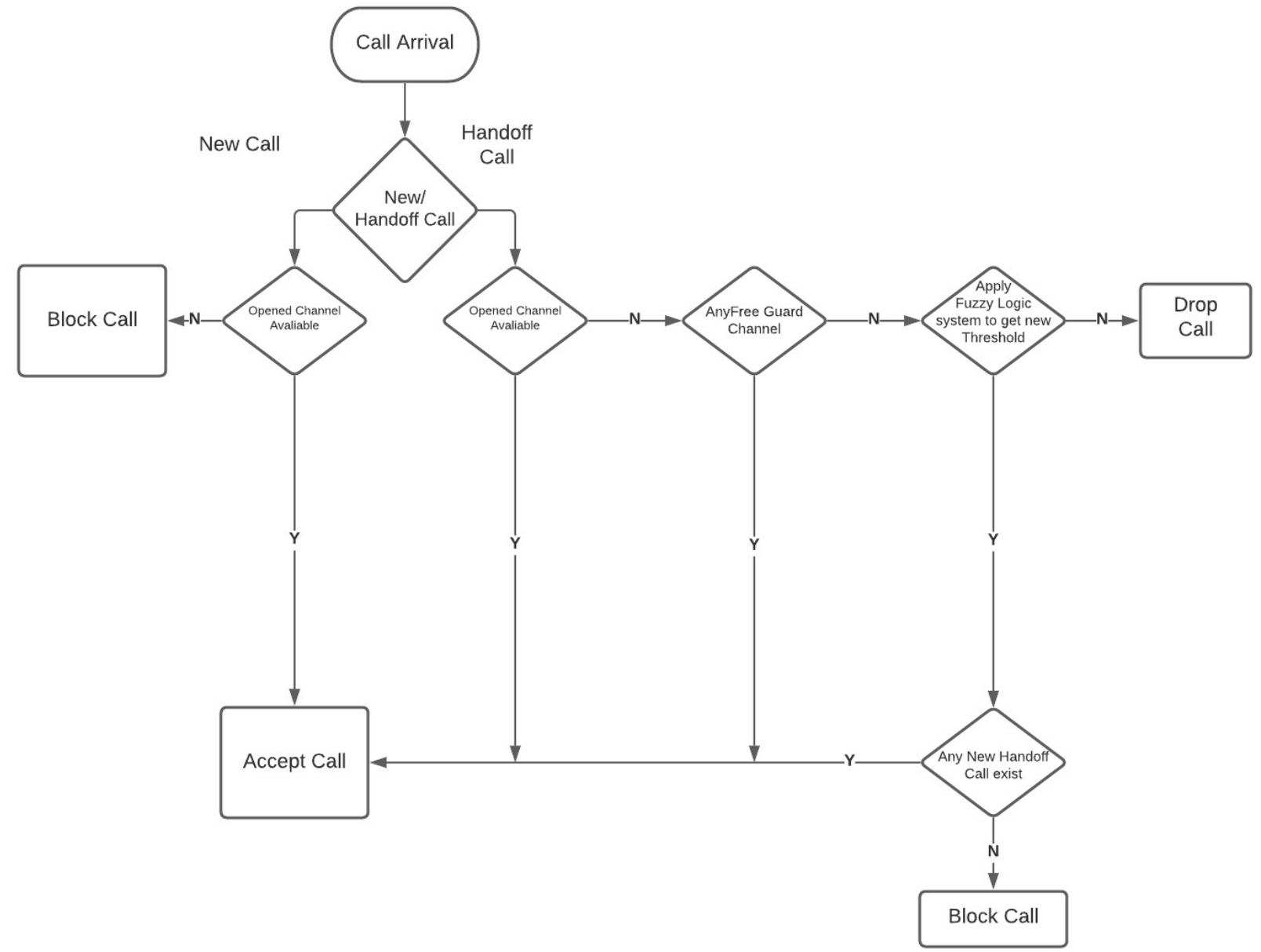

Figure 2: System Flowchart. 


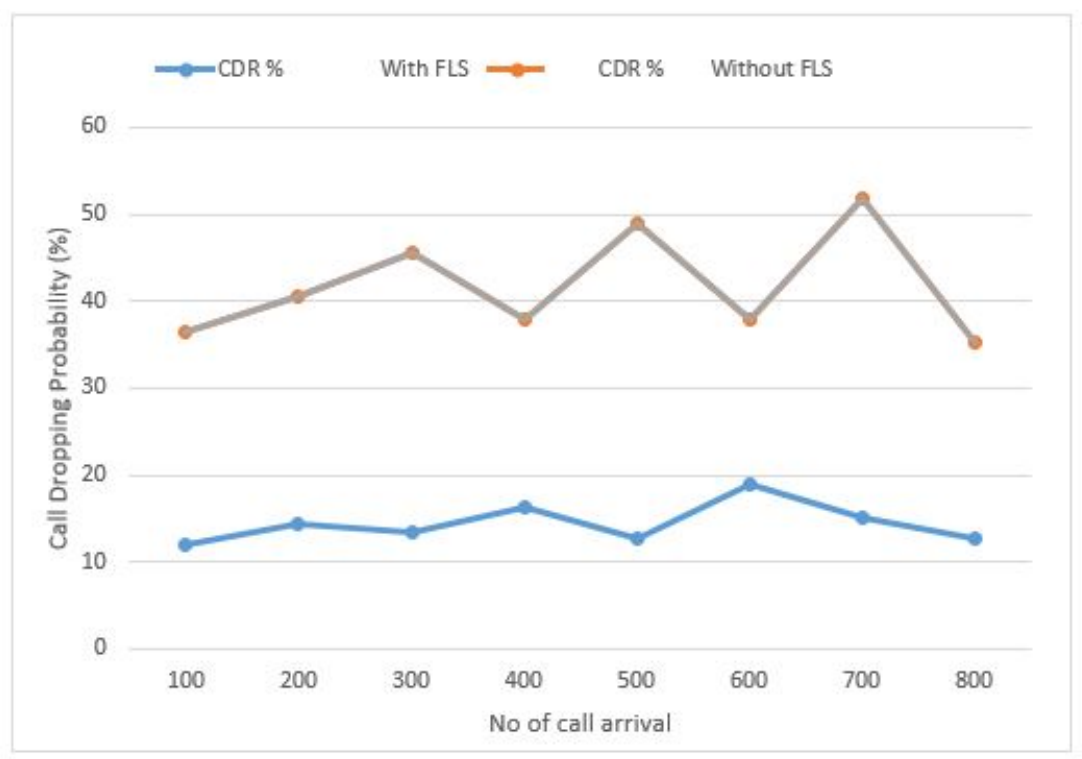

Figure 3: Graph of Percentage (\%) call dropping probability against call arrival.

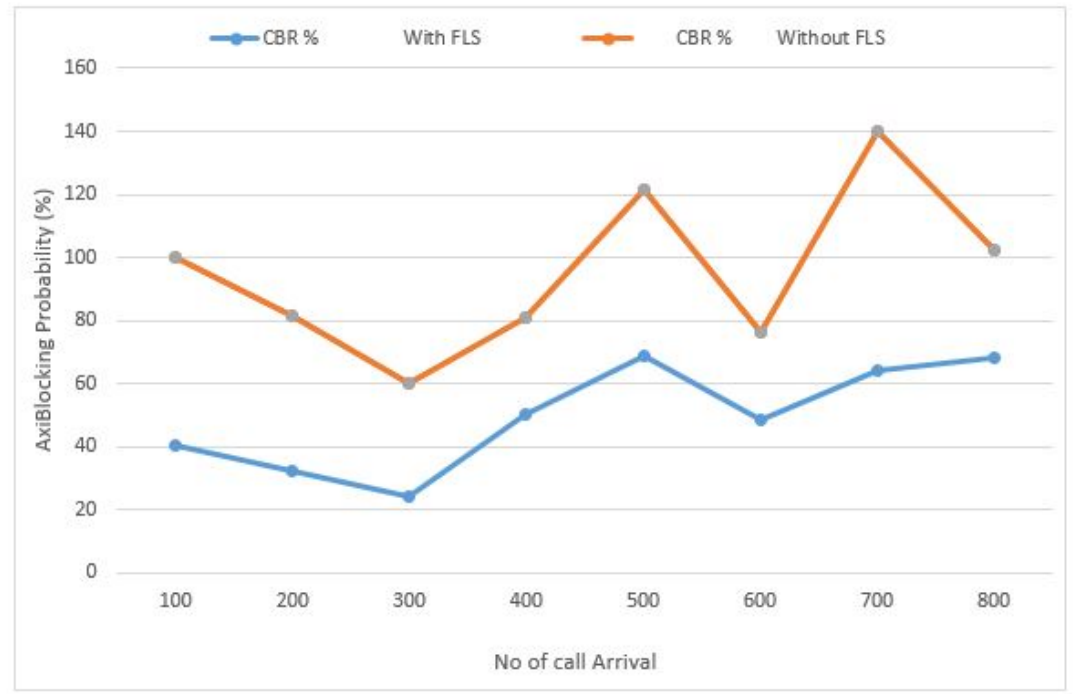

Figure 4: Graph of Percentage (\%) call blocking probability against call arrival.

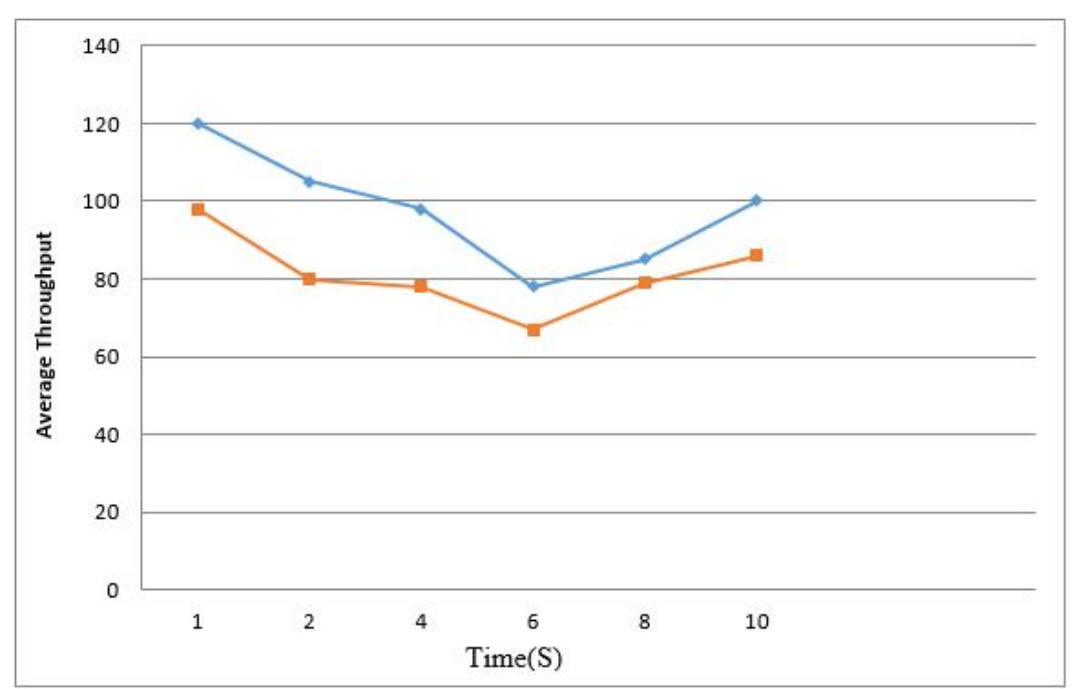

Figure 5: Throughput Graph. 
evaluation result in Fig. 4 shows the Call Blocking Rate (CBR) that applied fuzzy model, with the $\mathrm{CBR}$ values that range from 24.20 to 69.01 , while the CBR values without fuzzy logic model range from 28.22 to 75.65. These implies that the CBR of handoff calls decreases when fuzzy model was applied. The result provides the evidence of an improved performance in terms of reduced CBR with the application of fuzzy logic model. Thus, the proposed scheme with fuzzy logic model has proved to be more efficient than that of the ordinary guard channel scheme.

The model enhanced QoS further through the efficient utilization of channels reservation and channel allocation. There is increment in system performance as there is increase in the throughput of the system as shown in Fig. 5.

\section{CONCLUSION}

In this paper, the model made use of two different guard channel assignment schemes; one is fuzzy logic based while the other is without fuzzy logic. The guard channel is adjusted dynamically at various instances when the number of handoff calls in the guard channel exceed the threshold. Handoff call is given priority over the new call because termination of an established call frustrates users generally. The result shows that the scheme with fuzzy logic performed better as there was greater reduction in the call dropping probability compared to the call blocking probability.

\section{References}

[1] GSMA, “The world GSM coverage map," 2010.

[2] A. Edia, O. Osanaiye, F. Aina, and O. Ogundile, "Line start permanent magnet motors with doublebarrier configuration for magnet conservation and performance improvement," Comparison of Vertical Handover Decision-Based Techniques in Heterogeneous Networks, vol. 11, no. 9, pp. 239-259, 2018.

[3] G. Pankaj and D. Lobiyal, "Minimizing the cost of twotier cellular network withqueuing handoff calls in microcell using geneticalgorithm," Comparison of Vertical Handover Decision-Based Techniques in Heterogeneous Networks, vol. 5, no. 1, pp. 14-21, 2018.

[4] A. Edia, O. O., F. Aina, and O. Ogundile, "Comparison of vertical handover decision-based techniques in heterogeneous networks," International Journal of Communications, Network and System Sciences, vol. 11, pp. 239-259, 2018.

[5] A. P. Swati and P. Dhaval, "Dynamic channel allocation in mobile ad hoc network," International Journal of Current Engineering and and Scientific Research, vol. 2, no. 8, pp. 2394-2397, 2015.

[6] A. E. A. Suad and B. Amin, "Dynamic versus static channel allocation scheme," International Journal of Science and Research, vol. 4, no. 6, pp. 2338-2339, 2015.

[7] S. Malathy, G. Sudhasadasivam, K. Murugan, and S. Lokesh, "Adaptive slot allocation and bandwidth sharing for prioritized handoff calls in mobile networks," International Journal of Computer Science and Information Security, vol. 8, no. 1, pp. 52-57, 2010.

[8] S. Shivi and K. Arun, "Analysis of received signal strength under handoff condition using network simulator 2," International Journal of Computer Sciences and Engineering Open Access, vol. 6, no. 12, pp. 164$168,2018$.

[9] K. Jahangir, "Handover management in GSM cellular system," International Journal of Computer Applications, vol. 8, no. 12, pp. 14-24, 2010.
[10] J. Madhu and M. Ragini, "Adaptive call admission control and resource allocation in multi server wireless/cellular network," Journal of Industrial Engineering International, vol. 12, pp. 71-80, 2016.

[11] A. A. Adewale, S. N. John, and E. R. Adagunodo, "Performance Comparison of Dynamic Guard Channel Assignment with Buffered Prioritized Scheme for Mobile WiMAX Network," in Proceedings of the World Congress on Engineering, vol. 1, 2016.

[12] S. Alagu and T. Meyyappan, "Efficient utilization of channels using dynamic guard channel allocation with channel borrowing strategy in handoffs," Journal of Computer Science and Information Technology, vol. 2, pp. 235-244, 2012.

[13] S. Vikas, "Channel allocation scheme for cellular network using fuzzy logic," Asian Journal of Engineering and Applied Technology, vol. 7, no. 1, pp. 74-77, 2018.

[14] S. Shubham, K. Saurav, S. Hiralal, A. Anjali, and S. Jibendu, "An improved channel allocation scheme for mobile cellular network," Journal of Engineering (IOSRJEN), vol. 9, no. 4, pp. 26-31, 2019.

[15] P. Sangeetha and V. Bhanumathi, "Fuzzy-based dynamic time slot allocation for wireless body area networks," Sensors, vol. 19, pp. 1-20, 2019.

[16] O. Pharatlhatlhe, J. Daka, and G. E., "Cellular cluster channel allocation using an edge weight frequency assignment algorithm," Journal of Electrical and Electronic Systems, vol. 7, no. 3, pp. 1-5, 2018.

[17] A. Mohamed, B. A. Amin, G. Ashraf, and Q. M. Khalid, "Review of fixed channel assignment in mobile communication systems," International Journal of Advanced Engineering Research and Science, vol. 3, no. 12, pp. 128-132, 2016.

[18] C. Diego and H. Flávio, "Resource allocation algorithm for LTE networks using fuzzy based adaptive priority and effective bandwidth estimation," Journal of Wireless Networks, vol. 2, pp. 423-437, 2018. 